\title{
Update on mental health care in Lebanon
}

Sir,

In 2005-2007 we surveyed mental health services in Lebanon [1]. At the time, we found a paucity of community-based mental health services, deficiencies in education programmes for professionals and the public, misallocation of health care finances, and lack of a clear governmental policy on delivery of mental health services and prevention of mental illness. Several barriers related to the government, patients, and physicians were identified, and solutions to address them, and to move Lebanon forward in meeting World Health Organization (WHO) recommendations for action on mental health, were proposed. Four years have now passed since our survey. While we have not conducted further formal investigations as to the state of mental health care in Lebanon at this time, we would like to provide some updated points.
From 2005-2008, the Lebanese population grew by approximately 400 000. In 2008, approximately LL 3.7 trillion (US\$ 2.45 billion) were spent on health care [2] compared to 3.5 trillion in 2005 [1]. Total expenditure on health was $8.8 \%$ of the gross domestic product (GDP) [2]. This accounted for $11.7 \%$ of governmental expenditure that year. The majority of health care expenditure was from the private sector rather than the government, with the former contributing $53.9 \%$ of total health expenditure and the latter 46.1\% [2]. Out-of-pocket expenditures constituted $11.7 \%$ of total health expenditure. Therefore, as compared to 3 years earlier, total government expenditure on health care increased, but private sector expenditure was greater. Private household expenditure has apparently declined [2].

Regarding the availability and cost of medications, shortages in essential medications such as antipsychotics and mood stabilizers continue to occur intermittently. The cost of some medications has significantly declined, particularly generic medications, whereas others have become more expensive. Previously reported costs were based on 2007 prices [1]. Updated costs of maintenance therapy for an episode of mania, depression or psychosis as of 2009 include: clomipramime LL 25200 (US\$ 16.8), lithium (Camcolit ${ }^{\circ}$ ) LL 252000 (US\$ 168.0) and generic lithium, LL 75373 (US\$ 50.25), chlorpromazine LL 53579 (US\$ 35.7), Prozac LL 232062 (US\$ 154.71), Depakote LL 198855 (US\$ 132.5) and Risperdal ${ }^{\circ}$ LL 804000 (US\$ 536.0).

It is hoped that in the next decade, significant steps towards meeting WHO recommendations for action on mental health are taken in Lebanon as outlined [1].

\section{References}

1. Chahine LM, Chemali Z. Mental health care in Lebanon: policy, plans, and programmes. Eastern Mediterranean Health Journal, 2009, 15(6):1596-1612.
2. National health accounts. Lebanon. World Health Organization [website] (http://www.emro.who.int/emrinfo/index. asp?Ctry=leb, Accessed 10 April 2010).

Lama M. Chahine

Neurological Institute, Cleveland Clinic, Cleveland, Ohio, United States of America (chahinl@ccf.org)

Zeina N. Chemali

Director of Neuropsychiatry, Brigham and Women's Hospital, Harvard Medical School, Boston, Massachusetts, United States of America 